\title{
Two-dimensional vortex quantum droplets get thick
}

\author{
Zeda $\operatorname{Lin}^{1, \S}$, Xiaoxi Xu ${ }^{1, \S}$, Zikang Chen $^{1}$, Ziteng Yan $^{1}$, Zhijie Mai ${ }^{2,3}$, Bin Liu* \\ ${ }^{1}$ School of Physics and Optoelectronic Engineering, Foshan University, Foshan 528000, China \\ 2 Department of Applied Physics, College of Electronic Engineering, \\ South China Agricultural University, Guangzhou 510642, China \\ 3 School of Mathematics, Statistics and Physics, Newcastle University, \\ Newcastle upon Tyne, United Kingdom, NE1 YRU and \\ $\S$ These authors contributed equally to this work.
}

\begin{abstract}
We study two-dimensional (2D) vortex quantum droplets (QDs) trapped by a thicker transverse confinement with $a_{\perp}>1 \mu \mathrm{m}$. Under this circumstance, the Lee-Huang-Yang (LHY) term should be described by its original form in the three-dimensional (3D) configuration. Previous studies have demonstrated that stable $2 \mathrm{D}$ vortex QDs can be supported by a thin transverse confinement with $a_{\perp} \ll 1 \mu \mathrm{m}$. In this case, the LHY term is described by a logarithm. Hence, two kinds of confinement features result in different mechanisms of the vortex QDs. The stabilities and characteristics of the vortex QDs must be re-identified. In the current system, we find that stable 2D vortex QDs can be supported with topological charge number up to at least 4. We reformulated their density profile, chemical potential and threshold norm for supporting the stable vortex QDs according to the new condition. Unlike the QDs under thin confinement, the QDs in the current system strongly repel each other because the LHY term features a higher-order repulsion than that of the thin confinement system. Moreover, elastic and inelastic collisions between two moving vortex QDs are studied throughout the paper. Two kinds of collisions can be characterized by exerting different values of related speed. The dynamics of the stable nested vortex QD, which is constructed by embedding one vortex $\mathrm{QD}$ with a smaller topological number into another vortex $\mathrm{QD}$ with a larger number of topological charge, can be supported by the system.

Keywords: Lee-Huang-Yang correction,Quantum droplets, Thick confinement,Gross-Pitaevskii equation
\end{abstract}

\section{INTRODUCTION}

Recently, a novel type of quantum liquid self-bound state, referred to as quantum droplets (QDs), has been formed with the help of the zero-point quantum fluctuations of the collective Bogoliugov mode, which can be described theoretically by the Lee-Huang-Yang (LHY) correction [1] in three-dimensional (3D) space. This correction describes a repulsive beyond mean-field (MF) force, which plays the role of a higher-order nonlinear repulsive term and can arrest the collapse of attractive Bose gases induced by the MF force. These attractive Bose gases are collapsing dipolar Bose-Einstein condensates with a strong attractive dipole-dipole interaction [2 -4] , and a collapsing Bose-Bose (BB) mixture when the interspecies attraction is stronger than the intraspecies repulsion [5]. Experiments have reported that self-bound QDs were formed by the dipolar Bose-Einstein condensates (BECs) of dysprosium, erbium [6 8 ], and the BB mixture of kalium [9 11].

In the case of the BB mixture, the LHY correction can manifest a different mechanism for the lower dimensional system [12 16]. In the two-dimensional (2D) system with thin confinement with $a_{\perp} \ll 1 \mu m$, the energy of the LHY term is $\sim n^{2} \ln (n / \sqrt{e}$ ) (where $n$ dominates the density of the QD) [17 19] and gives rise to a term including a logarithm in the Gross-Pitaevskii equation (GPE). According to the behavior of the logarithm, the LHY term manifests repulsion and attraction when the QDs are under extremely dense and dilute conditions, respectively. The reduction of the BB mixture with the LHY corrections to the one-dimensional (1D) system is drastically different. Under this circumstance, the QDs are formed when the LHY term features only attraction, contrary to its repulsive sign in higher dimensions, while the total effect of the cubic mean-field force is tuned to repulsion, which competes with the LHY-induced attraction 20 22]. Recent studies have revealed that the LHY term in the lower dimensional systems can stabilize the QD embedded with vortices. It has been reported that vortex QDs can be stabilized up to at least $S=5$ in a 2D thin confinement system [23], and semidiscrete vortex QD, which can also be stabilized up to at least $S=5$, can be created in arrays of the coupled 1D confinement system 24]. In contrast with these results, vortex QDs in single-component dipolar condensates were found to be unstable 25]. However, for the case of 2D vortex QDs in the $\mathrm{BB}$ mixture, the stable $2 \mathrm{D}$ vortex QDs are created based on the condition of thin confinement in the transverse

*Electronic address: binliu@fosu.edu.cn 
direction (i.e., $\left.a_{\perp} \ll 1 \mu m\right)$. As mentioned above, the LHY term contains a logarithm under this circumstance. If the transverse confinement thickens (i.e., $a_{\perp}>1 \mu \mathrm{m}$ ), which is also the natural scale for BEC confinement in the 2D configuration, the LHY term may retreat to its original form, which is the same as the form in the $3 \mathrm{D}$ configuration (i.e., a quartic term [5, 26]). In this case, the LHY term manifests only strong repulsion, which is different from the effect of the logarithm in the case of thin transverse confinement. It is necessary to point out that stabilization of a vortex QD for the BB mixture in the full 3D configuration remains a challenging issue [27]. Because the LHY term plays an important role in stabilizing the vortex QD, how the change of the LHY term affects the stabilities and characteristics of the vortex QD is worth exploring.

In this paper, we aim to reconsider the stabilities and characteristics of $2 \mathrm{D}$ vortex QDs under thicker transverse confinement with the value of $a_{\perp}$ within a few microns. Under this circumstance, the system can still be termed as a quasi-2D one. The LHY term should be replaced by a quartic term in the GPE, which is the same as its original form in the 3D configuration. The rest of this paper is structured as follows: the model for the current system is described in Section II, and results of the 2D vortex QD in the new condition are discussed in Section III, and this work is concluded in Section IV.

\section{THE MODEL}

According to the ref. $([28,29])$, the underlying 3D GP equation supplemented by the LHY-induced quartic selfrepulsion term can be written, in the scaled form, as

$$
i \frac{\partial}{\partial t} \Phi=-\frac{1}{2} \nabla_{3 D}^{2} \Phi-g|\Phi|^{2} \Phi+|\Phi|^{3} \Phi+V(z) \Phi,
$$

where $\Phi$ stands for equal wave functions of two components of the BECs, and $V(z)=z^{2} /\left(2 a_{\perp}^{4}\right)$ is the transverse confinement. Then, the 3D to 2D reduction is performed by means of the standard substitution, as follows,

$$
\Phi(x, y, z, t)=\Psi(x, y, t) \exp \left(-\frac{1}{2 a_{\perp}^{2}} z^{2}-i \frac{t}{2 a_{\perp}^{2}}\right) .
$$

Substituting the solution Eq. (2) into Eq. (11), and followed by the averaging of Eq. (1) in the transverse direction, one yields

$$
i \frac{\partial \Psi}{\partial t}=-\frac{1}{2} \nabla_{2 D}^{2} \Psi-\frac{g}{\sqrt{3}}|\Psi|^{2} \Psi+\frac{1}{2}|\Psi|^{3} \Psi
$$

Further, with the help of additional rescaling, $(x, y) \rightarrow \sqrt{2}(x, y), t \rightarrow 2 t, g \rightarrow \frac{\sqrt{3}}{2} g$, the effective 2D GPE can be written as follows:

$$
i \frac{\partial \Psi}{\partial t}=-\frac{1}{2} \nabla_{2 D}^{2} \Psi-g|\Psi|^{2} \Psi+|\Psi|^{3} \Psi .
$$

Where $g>0$ is the strength of the self-attractive cubic nonlinearity (the intercomponent attraction being slightly stronger than the repulsion for each component [5]). The total norm under the symmetry condition can be characterized as

$$
N=\iint|\Psi(\mathbf{r})|^{2} d x d y
$$

and the Hamiltonian (energy) corresponding to Eq. (3) is

$$
E=\frac{1}{2} \iint\left[|\nabla \Psi|^{2}-g|\Psi|^{4}+\frac{4}{5}|\Psi|^{5}\right] d x d y .
$$

The objective of this work is to make use of the LHY effect for the stabilization of the vortex QDs in the present system. Thus the stationary QDs solutions with topological charge number $S=1,2, \cdots$ in the polar coordinates are looked for

$$
\Psi(\mathbf{r}, t)=\phi(r) \exp (-i \mu t+i S \theta)
$$


Substituting the solution Eq. (6) into Eq. (3), the real amplitude function $\phi(r)$ obeys a radial equation

$$
\mu \phi=-\frac{1}{2}\left(\frac{d^{2}}{d r^{2}}+\frac{1}{r} \frac{d}{d r}-\frac{S^{2}}{r}\right) \phi-g \phi^{3}+\phi^{4} .
$$

The stability of the stationary solution is analyzed by means of the linearized Bogoliugov-de Gennes (BdG) equations for perturbed wave functions, taken as

$$
\Psi(\mathbf{r}, t)=\left[\phi(r)+w(r) e^{-i \lambda t+i m \theta}+v^{*}(r) e^{i \lambda^{*} t-i m \theta}\right] e^{-i \mu t+i S \theta},
$$

where $w, v$, and $\lambda$ are eigenmodes and the instability growth rate corresponding to an integer azimuthal index $m$ of the perturbation. The linearization around the stationary solution leads to equations

$$
\begin{aligned}
& \lambda w=-\frac{1}{2}\left[\frac{d^{2}}{d r^{2}}+\frac{1}{r} \frac{d}{d r}-\frac{(S+m)^{2}}{r^{2}}\right] w+\left(-2 g \phi^{2}+\frac{5}{2} \phi^{3}-\mu\right) w+\left(-g \phi^{2}+\frac{3}{2} \phi^{3}\right) v, \\
& \lambda v=\frac{1}{2}\left[\frac{d^{2}}{d r^{2}}+\frac{1}{r} \frac{d}{d r}-\frac{(S-m)^{2}}{r^{2}}\right] v-\left(-2 g \phi^{2}+\frac{5}{2} \phi^{3}-\mu\right) v-\left(-g \phi^{2}+\frac{3}{2} \phi^{3}\right) w .
\end{aligned}
$$

Numerical solution of the linearized equations produces a spectrum of eigenfrequencies $\lambda$, the stability condition being that the spectrum of $\lambda$ must be real for at least $m=0,1,2,3$ [30, 31]. Moreover, the stability of the stationary solutions are also verified by direct simulations of the perturbed evolution in the framework of Eq. (3).

\section{RESULTS AND DISCUSSION}

\section{A. Stationary solutions}

Stationary solutions for Eq. (3) are numerically solved by the imaginary time method [32, 33]. Stable vortex QDs in this 2D system with thicker transverse confinement are found when the topological charge $S=1,2,3$, and 4 . Typical examples of the density pattern as well as the phase diagram of these vortex QDs for $g=1$ are displayed in Fig. 1. In Fig. 2, we give the direct simulations of the perturbed evolution results of $S=1$ and $S=4$, respectively, as well as the perturbation eigenvalues for the corresponding vortex QDs with $S=1$ and $S=4$ with different azimuthal index $m$. These results demonstrate that the vortex QDs can be stable at least up to $S=4$. Further, Figs. 33(a-d) show the density pattern of vortex QDs of $S=1$ with different values of $N$, which indicates that the vortex QDs in this system are also flat-top for sufficiently large Norm value.

To study the characteristics of the vortex QDs, we define the effective area for the QDs as

$$
A_{\mathrm{eff}}=\frac{\left(\iint|\Psi|^{2} d x d y\right)^{2}}{\iint|\Psi|^{4} d x d y},
$$

The functions of $A_{\text {eff }}$ for the vortex QDs with $(S, g)=(1,1)$ are shown in Fig. [3(e), which indicates that the curve for $A_{\text {eff }}(N)$ expands linearly with the increase of the total norm. The curve of $A_{\text {eff }}(N)$ can be linearly fitted by $A_{\text {eff }}=125+1.5 N$. In Fig. [3(f), the energy of the vortex QDs with $S=1$ as a function of $N$ are given, which shows that $E$ decrease linearly with the increase of $N$.

The influences of the total norm on other characteristics of the vortex QDs are also studied. Figs. 廿4(a,b) display the peak value, $|\phi|_{\max }^{2}$, and the chemical potential, $\mu$, as the functions of $N$. The function $|\phi|_{\max }^{2}(N)$ manifests that the curve of the function saturates to a fixed value for a sufficiently large value of $N$. Furthermore, the function of $\mu(N)$ satisfies the Vakhitov-Kolokolov (VK) criterion, i.e., $d \mu / d N<0$, which is a necessary stability condition for self-trapping modes in the attractive media 34 .

Similar to the case of vortex QDs in the thin confinement system, the numerical simulations also find a threshold norm, $N_{\text {th }}$, and the vortex QDs in the current system will be unstable if $N<N_{\text {th }}$. In Figs. 廿4(b), the blue curves represent vortex QDs with $S=1$ is dynamically stable when the norm values exceeding certain threshold $\left(N_{\text {th }} \approx 147\right)$. However, in the interval of $N<N_{\text {th }}$, a black short dashed curves, which represents the unstable vortex QDs, is also

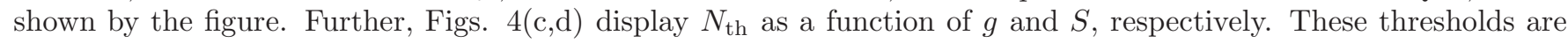
verified by computing the eigenvalues of $\lambda$, as well as the direct simulations. It is found that $N_{\operatorname{th}}(g)($ for $S=1)$ and $N_{\mathrm{th}}(S)$ (for $g=1$ ) are satisfied, respectively, as

$$
N_{\mathrm{th}}^{(S=1)}(g)=\alpha / g, \quad N_{\mathrm{th}}^{(g=1)}(S)=\alpha S^{2} .
$$


(a)

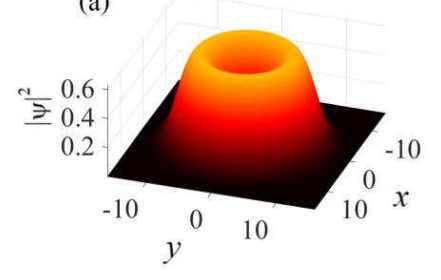

(b)
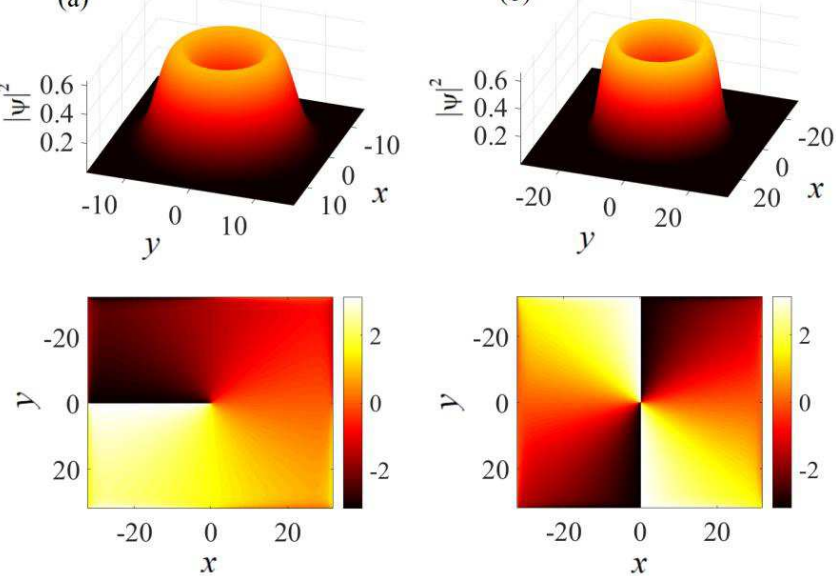

(e)

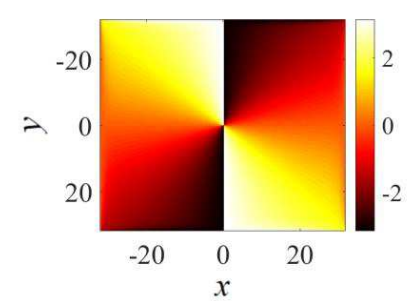

(f) (c)
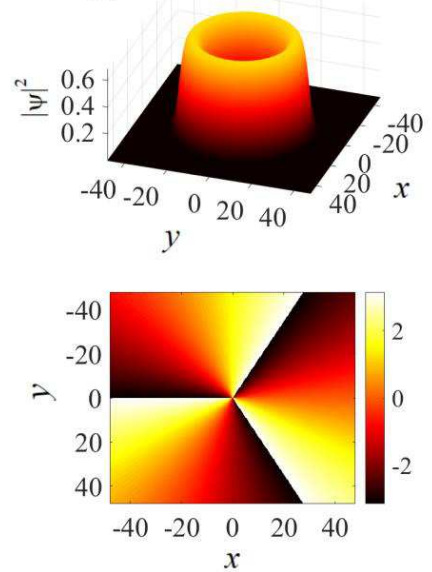

(g) (d)

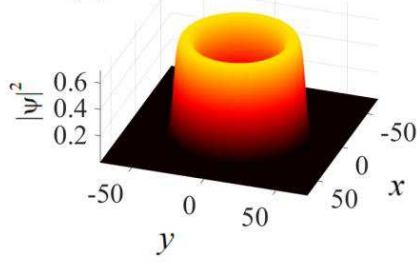

FIG. 1: Typical examples of stable vortex QDs with $S=1$ to 4 (from left to right). (a-d) Density patterns of the vortex QDs with $(N, S)=(200,1),(N, S)=(500,2),(N, S)=(1200,3)$, and $(N, S)=(2500,4)$. (e-h) The corresponding phase diagrams of the vortex QDs are in panels (a-d), respectively.
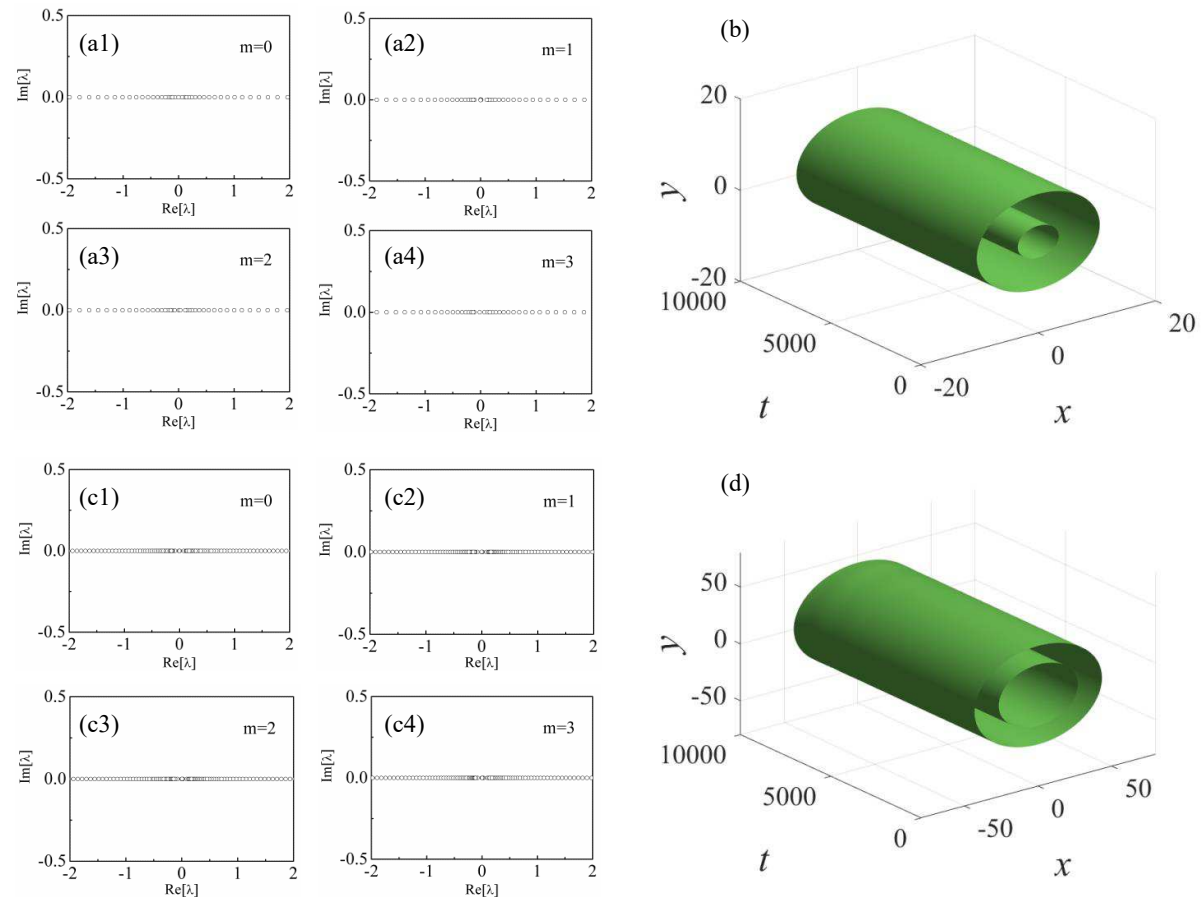

FIG. 2: Perturbation eigenvalues for the corresponding vortex QDs with $S=1$ and $N=200$ for different azimuthal index $m$ [in panels (a1)-(a4)] and direct simulations of the perturbed evolution of $\Psi$ [in panel (b)] are displayed, respectively. Perturbation eigenvalues for the corresponding vortex QDs with $S=4$ and $N=2500$ for different azimuthal index $m$ [in panels (c1)-(c4)] and direct simulations of the perturbed evolution of $\Psi$ [in panel (d)] are displayed, respectively.

The numerical simulation demonstrates that $\alpha \approx 147$, which can be identified by means of the threshold norm at $(S, g)=(1,1)$. According to our results, the smallest diameter for the vortex QDs, which is $(N, S)=(147,1)$, is $\sim 20$. If we assume the confinement is $2 \mu m$, according to the above transformations, $x=1 \sim \sqrt{2} \times 2 \mu m \approx 2.8 \mu m$. Therefore, the real size for this vortex droplets is $\sim 60 \mu \mathrm{m}$. If we want to let the $2 \mathrm{D}$ solution is relevant in the $3 \mathrm{D}$ system, " $a_{\perp}$ " should be at least expand to $>10 \mu \mathrm{m}$. It is necessary to piont out that the threshold $N_{t h}(S)$ for the vortex QDs in the current system is larger than their counterparts in model of thin confinement in Ref. [23], which indicates that 

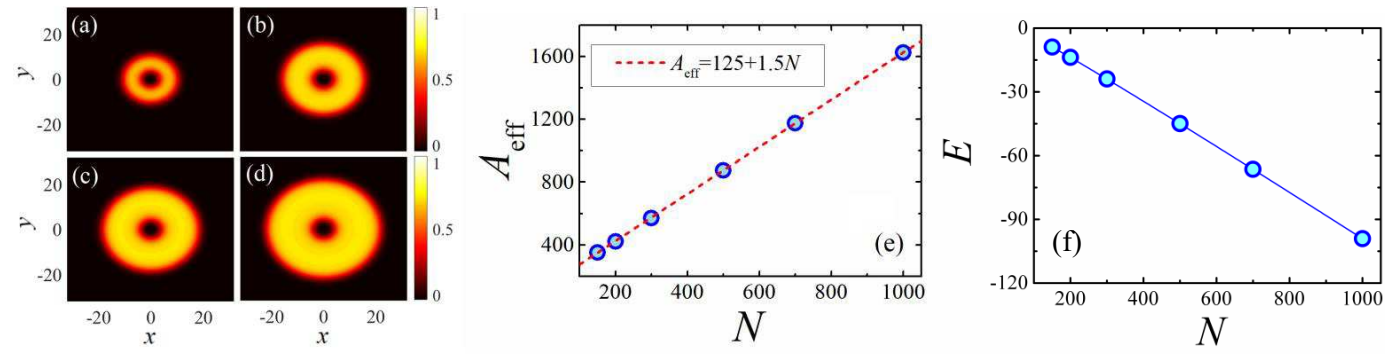

FIG. 3: (a-d) Typical examples of stable vortex QDs with $(N, S)=(200,1),(500,1),(700,1)$, and (1000,1), respectively. (e) The effective area of the vortex QDs with $S=1$ as a function of $N$. (f) The energy of the vortex QDs with $S=1$ as a function of $N$.

the stability area of the vortex QDs for the thin confinement system is larger than the thick confinement. Therefore, the vortex QDs in the model of thin confinement should be more stable than the current system.

In the following subsection, we will provide the theoretical analysis with respect to some of the numerical results in this subsection.
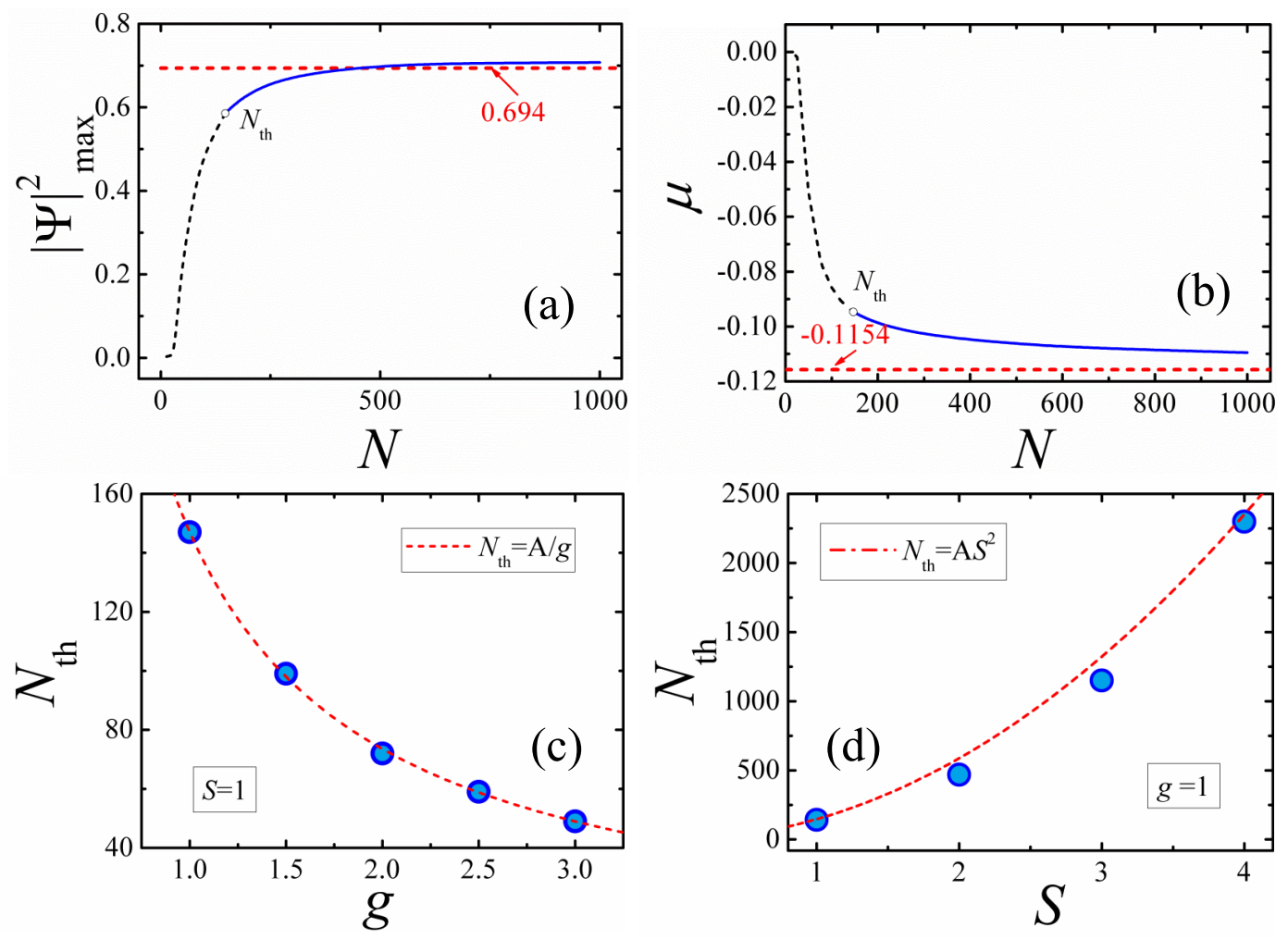

FIG. 4: (a) Peak density of the vortex QD, $|\Psi|_{\max }^{2}$, versus $N$. (b) Chemical potential of the vortex QDs as a function of $N$. In panels $(\mathrm{a}, \mathrm{b})$, we select $(g, S)=(1,1)$. (c) Threshold value of total norm for vortex QD with $S=1$ as a function of $g$. (d) Threshold value of total norm for vortex QD with $g=1$ versus topological charge number $S$.

\section{B. Analysis}

Some analyses based on the the characteristics of the vortex QDs are conducted as follow: because the vortex QDs generally have flat-top density profiles, one can therefore apply the Thomas-Fermi (TF) approximation to analyze 
them, i.e., neglecting the contribution from the kinetic term. Therefore, the energy density of the soliton can be written as

$$
\epsilon\left(n_{\max }\right)=-\frac{g}{2} n_{\max }^{2}+\frac{2}{5} n_{\max }^{5 / 2}
$$

where $n_{\max }=|\Psi|_{\max }^{2}$ is the peak value of the density profile. The value of $n_{\max }$ is determined by the minimization of the total energy. If the radius of the flat-top soliton is $R$, the total norm is

$$
N \approx A_{\mathrm{eff}} n_{\mathrm{max}}
$$

hence the area of the soliton can be written as $A_{\mathrm{eff}} \approx N / n_{\max }$ (this equation partially explains why the function of effective area versus $N$, i.e., $A_{\text {eff }}(N)$, can be linearly fitted) and, accordingly, the total energy is

$$
E \approx A_{\mathrm{eff}} \epsilon\left(n_{\max }\right) \approx N\left(-\frac{g}{2} n_{\max }+\frac{2}{5} n_{\max }^{3 / 2}\right) .
$$

This relationship explains the reason of $E$ linearly depends on $N$ in Fig. $3(f)$. Finally, the system selects the value of $n_{\max }$ which minimizes the total energy for fixed $N: d E / d n_{\max }=0$, hence

$$
n_{\max }=\left(\frac{5}{6} g\right)^{2} .
$$

If $g=1$, one may obtain $n_{\max }=|\Phi|_{\max }^{2} \approx 0.694$, which is in accordance with the magnitude of $|\Psi|_{\max }^{2}$ if the limit of $N$ is sufficiently large in Fig. 4(a). According to the above analysis,

$$
\text { when } \quad N \rightarrow \infty, \quad \mu \rightarrow-\left(\frac{5}{6} g\right)^{2} g+\left(\frac{5}{6} g\right)^{3} .
$$

For $g=1$, one may obtain $\mu \approx-0.1154$. In Fig. $4(\mathrm{~b})$, we can see that the $\mu(N)$ curve also trends to this limit.

The same results for $n_{\max }$ and the respective value $\mu\left(n_{\max }\right)$ can also be found in a different way. To this end, note that in the limit of very broad solitons, radial equation (7) becomes quasi-one-dimensional

$$
\mu \phi=-\frac{1}{2} \frac{d^{2} \phi}{d r^{2}}-g \phi^{3}+\phi^{4} .
$$

This equation can be derived from a formal Hamiltonian (if $r$ is formally treated as time), which remains constant in the course of the evolution along $r$

$$
h=\frac{1}{2} \mu \phi^{2}+\frac{1}{4}\left(\frac{d \phi}{d r}\right)^{2}+\frac{g}{4} \phi^{4}-\frac{1}{5} \phi^{5} .
$$

For solitons, $\phi(r=\infty)=0$, and hence one should set $h=0$ in Eq. (18).

In the limit of very broad solitons, the derivative terms in Eqs. (17) and (18) may be dropped, which yields an algebraic system

$$
\mu=-g \phi^{2}+\phi^{3}, \mu=-\frac{g}{2} \phi^{2}+\frac{2}{5} \phi^{3} .
$$

A solution of this system is identical to the values given by Eqs. (15) and (16).

\section{Dynamical process}

The dynamical process in this subsection is mainly focused on the interaction between two vortex QDs. Unlike the counterparts in the thin confinement system, which can merge with each other during the meeting between two QDs because the logarithm term can feature alternative attraction and repulsion, two QDs feature strong repulsion between each other because the LHY term manifests a quartic repulsion under the new condition. If the two vortex QDs close to each other (i.e., they have some overlap of their tails), they automatically repel each other. A typical example for this dynamic is displayed in Fig. [5(a). In this figure, two QDs automatically move away from each other because of the repulsion between them. The repulsive effect also influences the collision between two moving vortex QDs. If the 
(a)

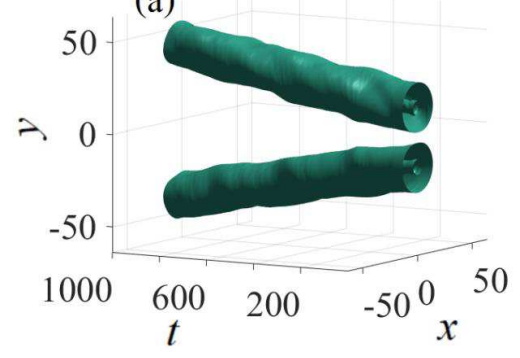

(b)

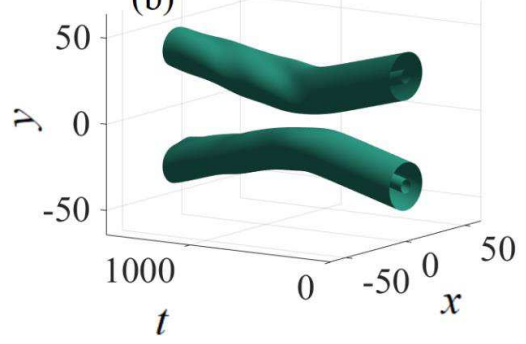

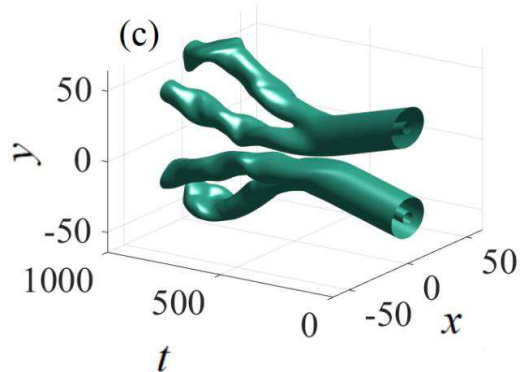

FIG. 5: Interactions between two vortex QDs. (a)Automatic repulsion. (b) Collision with small kick $(\eta=0.025)$, which creates a small relative velocity between them. (c) Collision with strong kick $(\eta=0.05)$, which gives rise to a large value of relative velocity between them. Here, the vortex QDs are select with $(N, S, g)=(200,1,1)$.

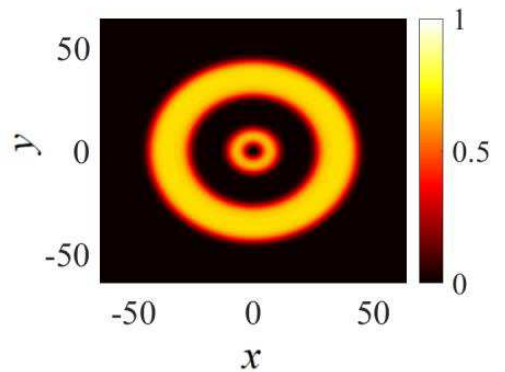

(a)

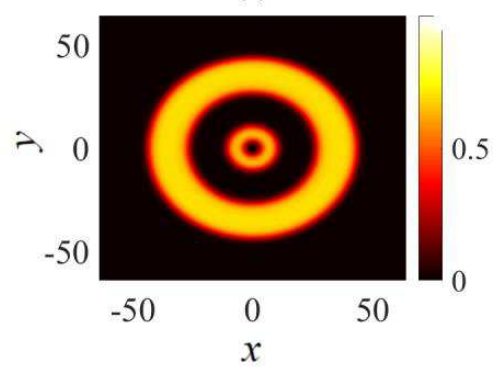

(d)

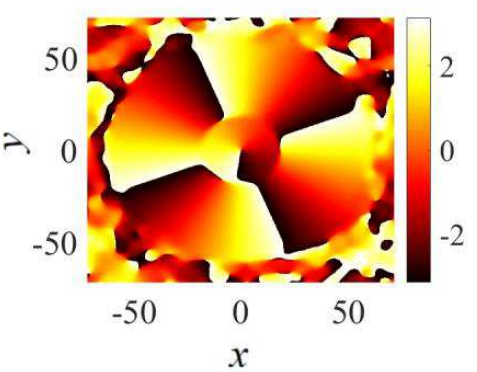

(b)

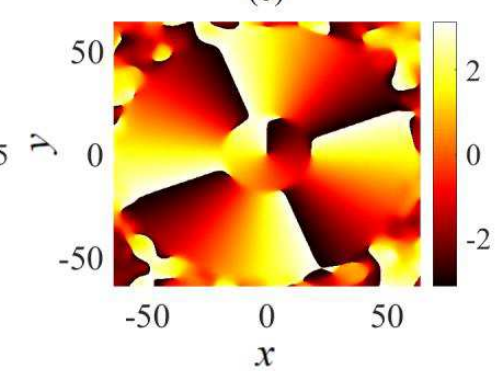

(e)

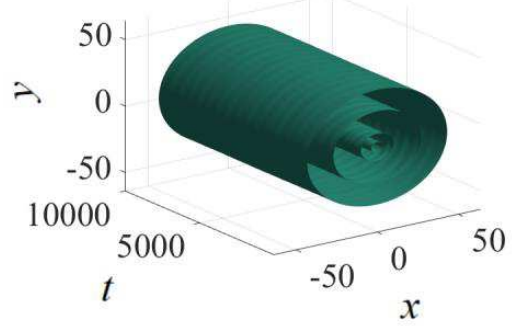

(c)

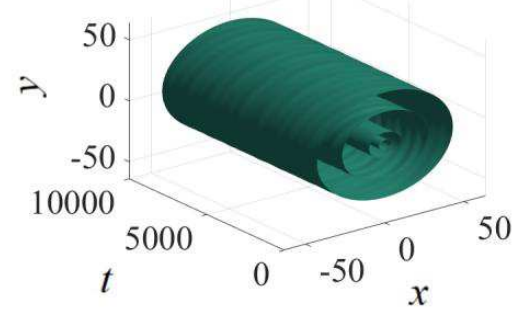

(f)

FIG. 6: Typical examples of the nested vortex QDs. The upper row includes a vortex QD with $(N, S, g)=(200,1,1)$ nests inside vortex QD with $(N, S, g)=(2500,4,1)$, while the lower row is the vortex QD with $(N, S, g)=(200,-1,1)$ nests inside a vortex QD with $(N, S, g)=(2500,4,1)$. (a,d)The output density pattern for the two nested vortex QDs at $t=10000$. (b,e)Output pattern of the phase structure for the two nested vortex QDs at $t=10000$. (c,f)Direct simulation of the two nested vortex QDs from $t=0 \rightarrow 10000$.

relative velocity between two colliding vortex QDs is small, an elastic collision occurs. In this case, two colliding QDs can retain their vortices after their collision. A typical example of an elastic collision between two moving vortex QDs is shown in Fig. [5(b). If the relative speed between them is large, an inelastic collision is expected. In this case, two stable vortex QDs are destroyed during their meeting. After the collision, they will separate into several fragments. A typical example of an inelastic collision of two vortex QDs with $S=1$ is shown in Fig. 5 (c).

Because the vortex QD with large topological charge number also has a large inner radius, a large empty space is created inside the QD. Hence, this space can be used to embed another QD with a smaller outer radius. If the outer ring of the embedded QD includes sufficient empty space adjacent to the inner ring of the encapsulated QD, these two QDs can stable coexist together for a sufficiently long period of time. Typical examples of such nested vortex QDs are displayed in Fig. 6. In the examples, two smaller vortex QDs with $(N, S)=(200, \pm 1)$ are embedded within larger vortex QDs with $(N, S)=(2500,4)$, respectively. The direct simulations show that these two nested vortex QDs can stablely coexist beyond $t=10^{4}$. During the realtime evolution, we can observe small turbulence created on their density profiles, however, such perturbation cannot destroy the stability of such a nested configuration. 


\section{CONCLUSION}

The objective of this work is to study the stabilities and characteristics of 2D vortex quantum droplets (QDs) formed by the Bose-Bose (BB) mixture under thicker transverse confinement with $a_{\perp}$ of up to at least a few microns. In this quasi-2D system, the LHY (Lee-Huang-Yang) term is replaced by its original form in the 3D configuration and features quartic repulsion. Stable 2D vortex QDs can be found in the current system up to at least $S=4$. Density profiles and the chemical potentials for the vortex QDs are systematically studied by numerical simulation and theoretical analysis throughout the paper. The threshold norms for supporting the stable vortex QDs are reformulated by the current system. Interactions between the vortex QDs are also considered. Unlike the QDs formed via the thin confinement system (i.e., $a_{\perp} \ll 1 \mu m$ ), the strong repulsion induced by the LHY term makes the vortex QDs repel each other in the current setting. Elastic and inelastic collisions between two moving vortex QDs are characterized by exerting different relative speeds. In the case of the elastic collision, the vortex QDs can maintain their vortices after the collision. In the case of inelastic collision, the vortex QDs are destroyed and split into fragments after the collision. Dynamics of the nested vortex QDs were also considered. This shows that the embedded QD and the encapsulated QD can stably coexist for sufficiently long duration of time if they have enough empty space between them. The result in this work may help to study the stabilization of vortex QDs formed by the BB mixture in the 3D configuration. Further, this work may help to study the stabilization of vortex QDs formed in optical lattice 22]. Specially, combining Abrikosov lattice with the current model maybe a very interesting generation, which may gain many novel phenomena to the QDs.

\section{Acknowledgments}

We appreciate the useful discussion from Prof. Yongyao Li. This work was supported by the NNSFC (China) through a grant Nos. 11905032, 11874112, the Key Research Projects of General Colleges in Guangdong Province through grant No. 2019KZDXM001, the Foundation for Distinguished Young Talents in Higher Education of Guangdong through grant No. 2018KQNCX279, and the Special Funds for the Cultivation of Guangdong College Students Scientific and Technological Innovation, No. xsjj202005zra01.

[1] Lee TD, Huang K, and Yang CN. Eigenvalues and Eigenfunctions of a Bose System of Hard Spheres and Its LowTemperature Properties. Phys Rev 1957;106:1135.

[2] Saito H. Path-Integral Monte Carlo Study on a Droplet of a Dipolar Bose-Einstein Condensate Stabilized by Quantum Fluctuation. J Phys Soc Jpn 2016;85:053001.

[3] Ferrier-Barbut I, Kadau H, Schmitt M, Wenzel M, and Pfau T. Observation of Quantum Droplets in a Strongly Dipolar Bose Gas. Phys Rev Lett 2015;116:215301.

[4] Boudjemâa A. Two-dimensional quantum droplets in dipolar Bose gases. New J Phys 2019;21:093027.

[5] Petrov DS. Quantum Mechanical Stabilization of a Collapsing Bose-Bose Mixture. Phys Rev Lett 2015;115:155302.

[6] Schmitt M, Wenzel M, Böttcher F, Ferrier-Barbut I, and Pfau T. Self-bound droplets of a dilute magnetic quantum liquid. Nature 2016;539:259.

[7] Chomaz L, Baier S, Petter D, Mark MJ, Wächtler F, Santos L, and Ferlaino F. Quantum-Fluctuation-Driven Crossover from a Dilute Bose-Einstein Condensate to a Macrodroplet in a Dipolar Quantum Fluid. Phys Rev X 2016;6:041039.

[8] Ferrier-Barbut I, Wenzel M, Böttcher F, Langen T, Isoard M, Stringari S, and Pfau T. Scissors Mode of Dipolar Quantum Droplets of Dysprosium Atoms. Phys Rev Lett 2018;120:160402.

[9] Cheiney P, Cabrera CR, Sanz J, Naylor B, Tanzi L, and Tarruell L. Bright Soliton to Quantum Droplet Transition in a Mixture of Bose-Einstein Condensates. Phys Rev Lett 2018;120:135301.

[10] Cabrera CR, Tanzi L, Sanz J, Naylor B, Thomas P, Cheiney P, Tarruell L. Quantum liquid droplets in a mixture of Bose-Einstein condensates. Science 2018;359:301.

[11] Semeghini G, Ferioli G, Masi L, Mazzinghi C, Wolswijk L, Minardi F, Modugno M, Modugno G, Inguscio M, and Fattori M. Phys Rev Lett 2018;120:235301.

[12] Petrov DS, and Astrakharchik GE. Ultradilute Low-Dimensional Liquids. Phys Rev Lett 2016;117:100401.

[13] Chiquillo E. Low-dimensional self-bound quantum Rabi-coupled bosonic droplets. Phys Rev A 2019;99:051601.

[14] Zin P, Pylak M, Wasak T, Gajda M, and Idziaszek Z. Quantum Bose-Bose droplets at a dimensional crossover. Phys Rev A 2018;98:051603(R).

[15] Ilg T, Kumlin J, Santos L, Petrov DS, and Büchler HP. Dimensional crossover for the beyond-mean-field correction in Bose gases. Phys Rev A 2018;98:051604(R).

[16] Chiquillo E. Equation of state of the one- and three-dimensional Bose-Bose gases. Phys Rev A 2018;97:063605.

[17] Kartashov YV, Malomed BA, and Torner L. Metastability of quantum droplet clusters. Phys Rev Lett 2019;122:193902. 
[18] Nilsson Tengstrand M, Stümer P, Karabulut EÖ, and Reimann SM. Rotating Binary Bose-Einstein Condensates and Vortex Clusters in Quantum Droplets. Phys Rev Lett 2019;123:160405.

[19] Li Y, Luo Z, Liu Y, Chen Z, Huang C, Fu S, Tan H, and Malomed BA. Two-dimensional solitons and quantum droplets supported by competing self- and cross-interactions in spin-orbit-coupled condensates. New J Phys 2017;19:113043.

[20] Astrakharchik GE, and Malomed BA. Dynamics of one-dimensional quantum droplets. Phys Rev A 2018;98:013631.

[21] Liu B, Zhang H, Zhong R, Zhang X, Qin X, Huang C, Li Y, Malomed BA. Symmetry breaking of quantum droplets in a dual-core trap. Phys Rev A 2019;99:053602.

[22] Zhou Z, Yu X, Zou Y, and Zhong H. Dynamics of quantum droplets in a one- dimensional optical lattice. Commun Nonlinear Sci Numer Simulat 2019;78:104881.

[23] Li Y, Chen Z, Luo Z, Huang C, Tan H, Pang W, and Malomed BA. Two-dimensional vortex quantum droplets. Phys Rev A 2018;98:063602.

[24] Zhang X, Xu X, Zheng Y, Chen Z, Liu B, Huang C, Malomed BA, and Li Y. Semidiscrete Quantum Droplets and Vortices. Phys Rev Lett 2019;123:133901.

[25] Cidrim A, Santos FEA, Henn EAL, and Macrí T. Vortices in self-bound dipolar droplets. Phys Rev A 2018;98:023618.

[26] Ferioli G, Semeghini G, Masi L, Giusti G, Modugno G. Collisions of Self-Bound Quantum Droplets. Phys Rev Lett 2019;122:090401.

[27] Kartashov YV, Malomed BA, Tarruell L, and Torner L. Three-dimensional droplets of swirling superfluids. Phys Rev A 2018;98:013612.

[28] Shamriz E, Chen Zh, Malomed BA, and Sakaguchi H. Singular Mean-Field States: A Brief Review of Recent Results. Condensed Matter 2010;5:20.

[29] Elad Shamriz, Zhaoping Chen, and Boris A. Malomed, Phys Rev A, in press, arXiv:2006.02576r1.

[30] Mihalache D, Mazilu D, Malomed BA, and Lederer F. Vortex stability in nearly-two-dimensional Bose-Einstein condensates with attraction. Phys Rev A 2006;73:043615.

[31] Dror N, Malomed BA. Symmetric and asymmetric solitons and vortices in linearly coupled two-dimensional waveguides with the cubic-quintic nonlinearity. Physica D 2011;240:526.

[32] Chiofalo LM, Succi S, and Tosi PM. Ground state of trapped interacting Bose-Einstein condensates by an explicit imaginary time algorithm. Phys Rev E 2000;62:7438.

[33] Yang J and Lakoba TI. Accelerated imaginary-time evolution methods for the computation of solitary waves. Stud Appl Math 2008;120:265.

[34] Vakhitov M and Kolokolov A. Stationary solutions of the wave equation in a medium with nonlinearity saturation. Radiophys. Quantum Electron 1973;16:783. 